\title{
Retinal Aging and Sirtuins
}

\author{
Yoko Ozawa ${ }^{a, b}$ Shunsuke Kubotaa, ${ }^{a}$ Toshio Narimatsu ${ }^{a, b}$ Kenya Yuki ${ }^{a, b}$ \\ Takashi Koto $^{a, b}$ Mariko Sasakia,b Kazuo Tsubota ${ }^{b}$ \\ a Laboratory of Retinal Cell Biology and ${ }^{b}$ Department of Ophthalmology, Keio University School of Medicine, \\ Tokyo, Japan
}

\section{Key Words}

Cell senescence $\cdot$ Apoptosis $\cdot$ Retinal aging $\cdot$ Sirtuin

\begin{abstract}
The process of aging involves the accumulating changes in the microenvironment that lead to cell senescence or apoptosis, and subsequent tissue or organ dysfunction. Multiple extrinsic and intrinsic events that cause DNA instability are associated with aging. Cells containing unstable DNA are biologically vulnerable, and if the DNA damage is too great for the cell to repair, it becomes senescent or dies by apoptosis. Thus, the cell's capacity to repair its DNA determines the progress of aging, at least in part. Here, we focus on the sirtuins, the mammalian homologs of the yeast life-span-extending molecule, Sir2. Among the sirtuin family proteins in mammals, the one most similar to yeast Sir2 is SIRT1, which is involved in multiple pathways, including the repair of DNA double-strand breaks. Although the role of SIRT1 in mammalian longevity is not clear, it is expressed throughout the retina, where it may suppress aging. In fact, a mutant mouse model of retinal degeneration shows an abnormal subcellular localization of SIRT1 protein and accelerated retinal cell apoptosis. Further analyses are required to elucidate the mechanism of DNA damage and repair, including the contributions of the sirtuins, in the aged or diseased retinas, which will help us understand the mechanisms of retinal aging.
\end{abstract}

Copyright $\odot 2010$ S. Karger AG, Basel
Accumulating changes in the microenvironment promote the aging of tissues and organs, which can eventually lead to their malfunction. The underlying mechanisms have long been explored, and their molecular basis is beginning to emerge. In this section, we describe how aging is evaluated scientifically and why sirtuins are an important topic in aging science. We also discuss the possible role of sirtuins in retinal aging.

\section{Aging of a Tissue/Organ and DNA Instability}

Aging research attracts the attention of researchers across various biological fields. Not only the length of the individual life span, but also the duration of tissue or organ function is of interest. In skin biology, the mechanism of 'photoaging', through which skin aging is accelerated by light exposure, has been widely investigated [1]. UV irradiation results in DNA damage in skin cells, which triggers DNA damage responses, including DNA repair. However, when the damage exceeds the ability of the cell to repair it, a cell cycle checkpoint response is triggered that leads to growth arrest and premature senescence, or to apoptosis, in order to prevent the genomic abnormality and instability from being inherited by other cells. In this case, aging is induced through an extrinsic mechanism.

On the other hand, telomeres, protective DNA structures at the ends of the chromosomes, are progressively

\section{KARGER \\ Fax +4161306 1234 E-Mail karger@karger.ch} www.karger.com
(C) 2010 S. Karger AG, Basel $0030-3747 / 10 / 0443-0199 \$ 26.00 / 0$

Accessible online at:

www.karger.com/ore
Yoko Ozawa, $\mathrm{MD}, \mathrm{PhD}$

Department of Ophthalmology, Keio University School of Medicine

35 Shinanomachi

Shinjuku-ku, Tokyo 160-8582 (Japan)

Tel. +81 33353 1211, Fax +8133359 8302, E-Mail yoko-o@ @c.itc.keio.ac.jp 
shortened during cell division, because the DNA replication machineries incompletely copy the 3 ' DNA ends, thereby inducing DNA loss [2]. Finally, this intrinsic mechanism causes the telomeres to shorten beyond a critical point that elicits a DNA damage response. This response causes irreversible cell cycle arrest (senescence), in cells that originally had normal genetic information.

Therefore, excessive DNA instability, induced by extrinsic or intrinsic events, to a level beyond its ability to be repaired, causes cell cycle arrest or apoptosis. As the number of senescent cells increases and that of normalfunctioning cells decreases, the tissue/organ becomes compromised and aged.

The retina is part of the central nervous system, and mature retinal cells are thought never to divide under normal conditions. Thus, cell cycle withdrawal occurs physiologically. Most neuronal cells in the brain do not proliferate either. Thus, decreases in functioning cells in the central nervous system may more easily lead to tissue/ organ dysfunction and aging than in other parts of the body $[3,4]$. Abnormal DNA is a pathological finding associated with some age-related neurodegenerative diseases, consistent with our understanding of aging as a consequence of DNA instability.

Various cytokines may also play a role in the aging process, again as a result of DNA instability [2]. In senescent human fibroblasts and epithelial cells, more than 40 distinct molecules can be secreted in response to DNA damage. Thus, chronic, not transient, changes in the microenvironment, due to the secretion of cytokines such as interleukins 6 or 8 , may be induced by DNA instability during tissue/organ aging.

\section{DNA Instability and Sirtuins}

Once DNA damage occurs, it triggers a series of downstream events, the purpose of which is to maintain and promote the survival of the tissue/organ as a whole $[5,6]$. First, cell proliferation is interrupted to allow time for DNA repair before synthesis. Then, if the repair is unsuccessful, processes to eliminate the cells are initiated.

Among the types of DNA damage, which include DNA single-strand breaks and base damage, DNA double-strand breaks are the hardest to repair, since both alleles are damaged simultaneously, leaving no complete template for the repair. In one of the main repair systems for double-strand breaks, nonhomologous end-joining, a

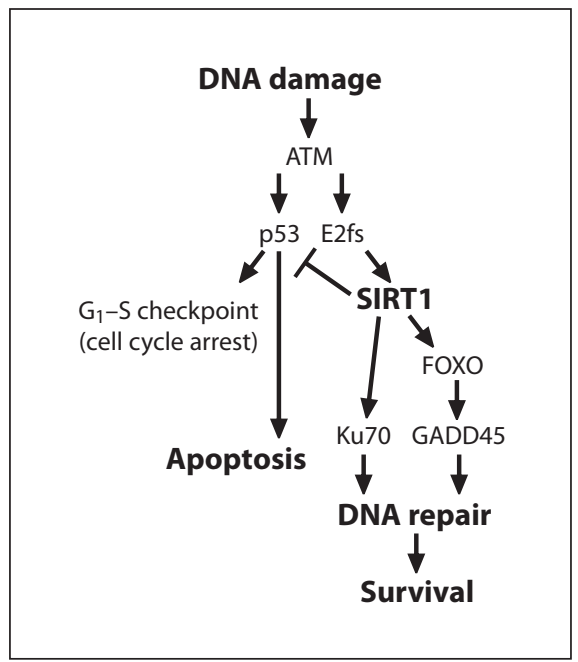

Fig. 1. SIRT1 in the DNA damage response. DNA damage (double-strand breaks) induces ataxia telangiectasia mutated (ATM), which activates p53 to slow the cell cycle (cell cycle checkpoints) to gain time for DNA repair. ATM also activates SIRT1 through E2fs, which in turn activates Ku70 (a protein for double-strand break repair), for the purpose of DNA repair and cell survival, and suppresses p53, to inhibit apoptosis. SIRT1 can also associate with forkhead transcription factor (FOXO) to induce antiapoptotic factors, such as manganese superoxide dismutase and growtharrest- and DNA-damage-inducible protein 45 (GADD45, a protein for DNA repair).

serine/threonine-specific protein kinase, ataxia telangiectasia mutated (ATM), is induced and activates p53 to stop the cell cycle at the $G_{1}$ phase, preventing it from entering the $S$ phase (fig. 1). Such a pause in cell cycle progression is termed a 'cell cycle checkpoint'. ATM activates the E2f transcription factors, which upregulate SIRT1. Deacetylation by SIRT1 activates Ku70, a nonhomologous end-joining DNA repair protein, while SIRT1 activity inhibits excessive activation of p53 and apoptosis. Therefore, SIRT1 regulates DNA stability and promotes cell survival.

SIRT1 is a mammalian homolog of yeast silent information regulator (Sir) 2, which plays a role in the life span extension by caloric restriction in yeast $[7,8]$. Sirtuins are class III histone deacetylases, which require nicotinamide adenine dinucleotide (NAD) as a cofactor. There are 7 mammalian Sir2 family members, designated SIRT1-SIRT7 [3, 9-12] (table 1), with SIRT1 being the most highly related to yeast $\operatorname{Sir} 2[3,9,13,14]$. The contribution of SIRT1/Sir2 to genome stability was shown in yeast cells and mouse embryonic stem cells [4]. SIRT1, 
Table 1. Mammalian sirtuins

\begin{tabular}{llll}
\hline $\begin{array}{l}\text { Human } \\
\text { sirtuin }\end{array}$ & $\begin{array}{l}\text { Subcellular } \\
\text { localization }\end{array}$ & Enzyme activity & Biology \\
\hline SIRT1 & Nucleus (cytosol) & Deacetylase & $\begin{array}{l}\text { DNA stability and cell proliferation (DNA } \\
\text { double-strand break repair) }\end{array}$ \\
\hline SIRT2 & Cytosol & Deacetylase & $\begin{array}{l}\text { Mitosis and cell differentiation (modulation } \\
\text { of microtubule network) }\end{array}$ \\
\hline SIRT3 & Mitochondria & Deacetylase & $\begin{array}{l}\text { Energy metabolism and responses to } \\
\text { oxidative stress (ATP level) }\end{array}$ \\
\hline SIRT4 & Mitochondria & ADP-ribosyltransferase & $\begin{array}{l}\text { Energy metabolism and responses to } \\
\text { oxidative stress (insulin secretion) }\end{array}$ \\
\hline SIRT5 & Mitochondria & Deacetylase & $\begin{array}{l}\text { Energy metabolism and responses to } \\
\text { oxidative stress }\end{array}$ \\
\hline SIRT6 & Nucleus & $\begin{array}{l}\text { ADP-ribosyltransferase, } \\
\text { deacetylase }\end{array}$ & $\begin{array}{l}\text { DNA stability and cell proliferation (DNA } \\
\text { single-strand break repair) }\end{array}$ \\
\hline SIRT7 & Nucleus & Deacetylase & \begin{tabular}{l} 
DNA stability and cell proliferation \\
\hline
\end{tabular} \\
\hline
\end{tabular}

like Sir2, modifies chromatin and silences the transcription of integrated reporter genes via histone deacetylation [15]. In SIRT1-deficient embryonic stem cells, oxidativestress-induced chromosomal fusions, which generally result from aberrantly repaired DNA, are clearly upregulated [4]. On the other hand, SIRT1 overexpression inhibits irradiation-induced cancers, by increasing DNA stability [4]. This result is particularly interesting in view of possible cancer therapies. However, the potential roles of SIRT1 in life span regulation have not yet been documented.

A biological role in aging was reported for SIRT6 [16]. In SIRT6-deficient mammalian cells (mouse embryonic fibroblasts and embryonic stem cells), proliferation is reduced, and sensitivity to DNA-damaging agents is increased, as is DNA instability. These events are caused by defective base excision repair, a repair mechanism for DNA single-strand breaks. The SIRT6-deficient cells show chromosomal aberrations and genome instability, even in the absence of artificial stimuli. Moreover, SIRT6deficient mice have a reduced life span. This may be due to direct impairment of the base excision repair system; however, these mice also show severely reduced insulinlike growth factor 1 and blood glucose levels, which might also promote DNA instability.

The deacetylase activity of SIRT1 has other substrates besides histones (fig. 1). It also deacetylates p53 (described above), nuclear factor $\kappa \mathrm{B}(\mathrm{NF}-\kappa \mathrm{B})$ and forkhead transcription factor (FOXO) [6]. Deacetylated NF- $\kappa \mathrm{B}$ is easily degraded and has less activity, which inhibits apop- tosis. FOXO, a downstream component of insulin signaling, can increase the transcription of both antiapoptotic factors, such as manganese superoxide dismutase (Mn-SOD), and a DNA repair factor, growth-arrest- and DNA-damage-inducible protein 45 (GADD45), and proapoptotic ones, such as B-cell-lymphoma-2-interacting mediator (BIM) and Fas ligand, depending on the context. These different effects can be explained, at least in part, by FOXO regulation by SIRT1. FOXO-induced apoptosis is decreased when SIRT1 is overexpressed in cerebellar neurons [17]. The deacetylation of FOXO by SIRT1 decreases the DNA-binding activity of FOXO and reduces the transcription of apoptotic factors [18]. On the other hand, the association of SIRT1 with FOXO upregulates the expression of antiapoptotic factors. Therefore, SIRT1 uses multiple pathways to modulate cellular resistance to genotoxic stress and apoptosis [13].

SIRT1 protein is mainly localized to the nucleus; however, it can be translocated to the cytoplasm in the presence of oxidative stress, leading to apoptosis [19]. This phenomenon is also observed in Caenorhabditis elegans [20]. Sir2.1 translocates from the nucleus to the cytoplasm as an early event of apoptosis, although the causal relationship and the kinetics of this translocation are still obscure.

Therefore, the sirtuins have critical roles in DNA stability and antiapoptotic processes, and they remain key molecules for aging research. 


\section{Sirtuins in the Retina}

SIRT1 is expressed throughout the retina, including the retinal ganglion cells, inner retinal cells, photoreceptor cells and retinal pigment epithelial cells [21]. SIRT1targeted mice show abnormal retinal development as early as embryonic day 12.5; these abnormalities include persistence of the optic fissure and disorganized retinal morphogenesis, with decreased cellularity in multiple retinal layers [22], although it is not yet known whether these embryonic retinal abnormalities are associated with an increase in p53 expression, as observed in SIRT1targeted mouse embryonic fibroblasts. The deletion of the upstream inducers of SIRT1, E2fs, also cause retinal cell death with the hyperacetylation (i.e. activation) of p53 [23].

SIRT1 activates multiple pathways and has been reported to function in animal models of inflammatory disease. In a multiple sclerosis model of experimental autoimmune encephalomyelitis, the intravitreal injection of SIRT1 activators prevented the death of retinal ganglion cells [24]. This effect was obtained without suppressing the infiltration of inflammatory cells into the optic nerve sheath, suggesting that SIRT1 acted in the retinal ganglion cells themselves.

This successful treatment of ganglion cells is consistent with the result of an experiment using explant neurons of the dorsal root ganglion [25]. Ganglion cell death after axotomy, called wallerian degeneration, is avoided by introduction of nicotinamide mononucleotide adenylyltransferase 1 (Nmnat1), an enzyme in the NAD biosynthesis pathway, which leads to an increase in nuclear NAD. SIRT1 activity induced by a polyphenol compound, resveratrol, has a similar effect. These results indicated that SIRT1, which is NAD dependent, is the effector of the ganglion cell protection conferred by the increased Nmnat1 activity and NAD. Moreover, Nmnat1 is normally expressed in retinal ganglion cells and their axons [26], which should enable SIRT1 activation in these cells.

A decrease in the deacetylation activity of SIRT1 during retinal inflammation was reported in the endotoxininduced uveitis model [27]. In this mouse model, 8-oxo2 -deoxyguanosine (8-OHdG), an oxidized form of deoxyguanosine, a component of DNA, is upregulated, and resveratrol suppresses this upregulation. It would be interesting to examine whether this reduction in DNA oxidation is related to an effect of SIRT1 on DNA and discuss the possible involvement of inflammation in aging.

\section{Role of Sirtuins in the Aging of the Retina}

Age-related DNA damage is reported in the rodent retina. An increase in oxidative DNA damage in the 2 -year-old retina compared to the 5-month-old retina was shown by 8 -OHdG labeling [28]. The authors concluded that this increase in labeling reflected mitochondrial DNA damage resulting from decreased levels of DNA repair enzymes. However, the role of sirtuins in this phenomenon is not addressed.

Abnormal SIRT1 expression is observed in a mutant mouse model of retinal degeneration ( $r d$ mice) that progresses with age; these $r d$ mice have a point mutation in the $\beta$-subunit of rod photoreceptor cGMP phosphodiesterase [21]. In the normal mouse retinal ganglion cells, inner nuclear layer cells and a subset of photoreceptors, thought to be cone cells, SIRT1 immunoreactivity appears as granular staining in the nuclei. In the rod photoreceptor cells, it appears as weak peripheral nuclear staining (but is still within the nucleus). However, in the $r d$ mice, SIRT1 in the rod photoreceptor cells translocates to the cytoplasm around postnatal day 15 , when apoptotic markers (caspase 12 or apoptosis-inducing factor (AIF) as well as transferase-mediated uridine nick endlabeling (TUNEL) staining) become apparent. This finding is consistent with another report that the translocation of SIRT1 protein from the nucleus to the cytoplasm precedes apoptosis in C. elegans [20]. The $r d$ mutants lose their photoreceptor cells precociously, which is considered an aging phenotype. A similar theory has been proposed to explain brain diseases, such as Parkinson's disease and Alzheimer's disease, in which brain cells degenerate earlier than in the normal life course. Further analyses are required to determine whether the translocation of SIRT1 from the nucleus leads to DNA instability in $r d$ mice and in other systems.

In summary, the aging of tissues/organs, including the retina, is caused by accumulated changes that lead to their dysfunction. The fundamental cause is believed to involve DNA instability, which may be induced by extrinsic stimuli, such as light exposure or inflammation, or by intrinsic factors, such as gene abnormalities. The ability of sirtuins to stabilize DNA may, at least in part, determine the progress of aging in the retina.

\section{Acknowledgement}

We thank Dr. Susumu Ishida for advice in preparing the manuscript. 


\section{References}

-1 Nakanishi M, Niida H, Murakami H, Shimada M: DNA damage responses in skin biology - implications in tumor prevention and aging acceleration. J Dermatol Sci 2009;56: 76-81.

2 Campisi J, Yaswen P: Aging and cancer cell biology, 2009. Aging Cell 2009;8:221-225.

3 Gan L, Mucke L: Paths of convergence: sirtuins in aging and neurodegeneration. Neuron 2008;58:10-14.

4 Oberdoerffer P, Michan S, McVay M, Mostoslavsky R, Vann J, Park SK, Hartlerode A, Stegmuller J, Hafner A, Loerch P, Wright SM, Mills KD, Bonni A, Yankner BA, Scully R, Prolla TA, Alt FW, Sinclair DA: Sirt1 redistribution on chromatin promotes genomic stability but alters gene expression during aging. Cell 2008;135:907-918.

5 Jackson SP: Sensing and repairing DNA double-strand breaks. Carcinogenesis 2002;23: 687-696.

-6 Niedernhofer LJ, Robbins PD: Signaling mechanisms involved in the response to genotoxic stress and regulating life span. Int J Biochem Cell Biol 2008;40:176-180.

$\checkmark 7$ Bass TM, Weinkove D, Houthoofd K, Gems D, Partridge L: Effects of resveratrol on lifespan in Drosophila melanogaster and Caenorhabditis elegans. Mech Ageing Dev 2007; 128:546-552.

$>8$ Guarente L, Kenyon C: Genetic pathways that regulate ageing in model organisms. Nature 2000;408:255-262.

-9 Finkel T, Deng CX, Mostoslavsky R: Recent progress in the biology and physiology of sirtuins. Nature 2009;460:587-591.

10 Frye RA: Phylogenetic classification of prokaryotic and eukaryotic Sir2-like proteins. Biochem Biophys Res Commun 2000;273: 793-798.

$\checkmark 11$ Haigis MC, Sinclair DA: Mammalian sirtuins: biological insights and disease relevance. Annu Rev Pathol 2010;5:253-295.
12 Haigis MC, Guarente LP: Mammalian sirtuins - emerging roles in physiology, aging, and calorie restriction. Genes Dev 2006;20: 2913-2921.

13 Blander G, Guarente L: The Sir2 family of protein deacetylases. Annu Rev Biochem 2004;73:417-435.

14 North BJ, Verdin E: Sirtuins: Sir2-related NAD-dependent protein deacetylases. Genome Biol 2004;5:224.

15 Vaquero A, Scher M, Lee D, Erdjument-Bromage $\mathrm{H}$, Tempst P, Reinberg D: Human SIRT1 interacts with histone $\mathrm{H} 1$ and promotes formation of facultative heterochromatin. Mol Cell 2004;16:93-105.

16 Mostoslavsky R, Chua KF, Lombard DB, Pang WW, Fischer MR, Gellon L, Liu P, Mostoslavsky G, Franco S, Murphy MM, Mills KD, Patel P, Hsu JT, Hong AL, Ford E, Cheng HL, Kennedy C, Nunez N, Bronson R, Frendewey D, Auerbach W, Valenzuela D, Karow M, Hottiger MO, Hursting S, Barrett JC, Guarente L, Mulligan R, Demple B, Yancopoulos GD, Alt FW: Genomic instability and aging-like phenotype in the absence of mammalian SIRT6. Cell 2006;124:315-329.

17 Brunet A, Sweeney LB, Sturgill JF, Chua KF, Greer PL, Lin Y, Tran H, Ross SE, Mostoslavsky R, Cohen HY, Hu LS, Cheng HL, Jedrychowski MP, Gygi SP, Sinclair DA, Alt FW, Greenberg ME: Stress-dependent regulation of FOXO transcription factors by the SIRT1 deacetylase. Science 2004;303:20112015.

18 Giannakou ME, Partridge L: The interaction between FOXO and SIRT1: tipping the balance towards survival. Trends Cell Biol 2004; 14:408-412.

19 Jin Q, Yan T, Ge X, Sun C, Shi X, Zhai Q: Cytoplasm-localized SIRT1 enhances apoptosis. J Cell Physiol 2007;213:88-97.

20 Greiss S, Hall J, Ahmed S, Gartner A: C. elegans SIR-2.1 translocation is linked to a proapoptotic pathway parallel to cep-1/p53 during DNA damage-induced apoptosis. Genes Dev 2008;22:2831-2842.
21 Jaliffa C, Ameqrane I, Dansault A, Leemput J, Vieira V, Lacassagne E, Provost A, Bigot K, Masson C, Menasche M, Abitbol M: Sirt1 involvement in rd10 mouse retinal degeneration. Invest Ophthalmol Vis Sci 2009;50: 3562-3572.

22 Cheng HL, Mostoslavsky R, Saito S, Manis JP, Gu Y, Patel P, Bronson R, Appella E, Alt FW, Chua KF: Developmental defects and p53 hyperacetylation in Sir2 homolog (SIRT1)-deficient mice. Proc Natl Acad Sci USA 2003;100:10794-10799.

23 Chen D, Pacal M, Wenzel P, Knoepfler PS, Leone G, Bremner R: Division and apoptosis of E2f-deficient retinal progenitors. Nature 2009;462:925-929.

-24 Shindler KS, Ventura E, Rex TS, Elliott P, Rostami A: SIRT1 activation confers neuroprotection in experimental optic neuritis. Invest Ophthalmol Vis Sci 2007;48:36023609

25 Araki T, Sasaki Y, Milbrandt J: Increased nuclear NAD biosynthesis and SIRT1 activation prevent axonal degeneration. Science 2004;305:1010-1013.

26 Kitaoka Y, Hayashi Y, Kumai T, Takeda H, Munemasa Y, Fujino H, Kitaoka Y, Ueno S, Sadun AA, Lam TT: Axonal and cell body protection by nicotinamide adenine dinucleotide in tumor necrosis factor-induced optic neuropathy. J Neuropathol Exp Neurol 2009; 68:915-927.

$>27$ Kubota S, Kurihara T, Mochimaru H, Satofuka S, Noda K, Ozawa Y, Oike Y, Ishida S, Tsubota K: Prevention of ocular inflammation in endotoxin-induced uveitis with resveratrol by inhibiting oxidative damage and nuclear factor-kappaB activation. Invest Ophthalmol Vis Sci 2009;50:3512-3519.

28 Wang AL, Lukas TJ, Yuan M, Neufeld AH: Age-related increase in mitochondrial DNA damage and loss of DNA repair capacity in the neural retina. Neurobiol Aging, E-pub ahead of print. 